\title{
Are glomerular filtration rate estimations necessary before high dose methotrexate?
}

\author{
H Rees, I M Hann, J M Chessells, D K H Webb
}

\begin{abstract}
Glomerular filtration rates (GFR) were estimated in 168 children (227 estimates) before treatment for haematological malignancies with high dose, intravenous methotrexate. Clinical management was altered on the basis of GFR in only two cases, both of whom had tumour lysis syndrome. Routine estimations of GFR do not contribute to management.

(Arch Dis Child 1999;81:339-340)
\end{abstract}

Keywords: methotrexate; glomerular filtration rate; haematological malignancies

High dose methotrexate (MTX) is a common component of treatment regimens for acute lymphoblastic leukaemia (ALL) and nonHodgkin's lymphoma (NHL). Methotrexate is renally excreted, ${ }^{1}$ and in the UK glomerular filtration rate (GFR) estimation is routinely stipulated before administration. However, this stipulation commonly causes logistic difficulties in treatment planning, may delay treatment, and may not be cost effective. Furthermore, high dose MTX is administered without routine estimation of GFR in several European countries. The experience with GFR estimation before administration of high dose MTX was reviewed in a single regional cancer centre.

\section{Methods}

The patient database at Great Ormond Street Hospital for Children (London, UK) was reviewed to identify children treated for ALL or NHL in the Medical Research Council (MRC) clinical trials UKALL XI and Infant ALL, and the United Kingdom Children's Cancer Study Group (UKCCSG) clinical trials LMB 9001-9004. Details of GFR estimations, plasma creatinine, and clinical history were obtained for all children scheduled to receive high dose MTX. The cost of GFR estimation was calculated on the basis of consumables and laboratory time, but did not include assessment of medical and nursing staff costs.

Haematology, Camelia

Botnar Laboratories,

Great Ormond Street

Hospital for Children,

London WC1N 3JH,

UK

H Rees

I M Hann

J M Chessells

D K H Webb

Correspondence to: Dr Webb.

Accepted 4 June 1999 for 11 children, and there was no record GFR estimate in 18. However, high dose MTX proceeded on schedule for these patients. Forty nine children had multiple estimations: 42 due to protocol stipulation, and seven for a previous abnormal result.

There were 16 results outside the normal range for our laboratory $(80-160 \mathrm{ml} / \mathrm{min} /$ $1.73 \mathrm{~m}^{2}$ ). One child had a GFR $>400 \mathrm{ml} / \mathrm{min} /$ $1.73 \mathrm{~m}^{2}$ owing to a technical error, and a repeat GFR was normal. One patient had a repeat GFR because of delay in clearance of high dose MTX requiring folinic acid rescue for 96 hours after an initial normal estimate. The repeat showed a value of $67 \mathrm{ml} / \mathrm{min} / 1.73 \mathrm{~m}^{2}$, treatment was not modified, the child cleared subsequent high dose MTX satisfactorily, and no further estimations were made. The remaining 14 children had a low initial GFR.

Seven children had initial values from 70 to $79 \mathrm{ml} / \mathrm{min} / 1.73 \mathrm{~m}^{2}$ but treatment continued without modification or delay. Two had repeat estimations two and four weeks later, which were normal. Seven children had an initial GFR $<70 \mathrm{ml} / \mathrm{min} / 1.73 \mathrm{~m}^{2}$, but treatment was modified in only two cases. One child with infant ALL had a GFR of $47 \mathrm{ml} / \mathrm{min} / 1.73 \mathrm{~m}^{2}$ after tumour lysis syndrome during induction chemotherapy, as well as severe sepsis requiring admission to an intensive care unit and treatment with multiple nephrotoxic antibiotics and amphotericin B after the first intensification block. High dose MTX was delayed by two weeks until the GFR had improved and further assessment after one month confirmed a normal value. The second child, with $\mathrm{B}$ cell NHL, also had tumour lysis syndrome during induction requiring dialysis. The GFR was $68 \mathrm{ml} / \mathrm{min} / 1.73 \mathrm{~m}^{2}$; a repeat GFR estimation after one week was normal, and high dose MTX was administered after a delay of two weeks.

Calculated GFR estimation costs for the whole group were $£ 9500$.

\section{Discussion}

We conclude that routine GFR estimation is unnecessary in most children treated with high dose MTX for ALL and NHL-in our series, estimation changed management in only two cases who were able to proceed with planned treatment in full doses after a short delay for improvement in renal function. Both of these children had experienced tumour lysis syndrome, and one had required intensive care and multiple nephrotoxic drugs including amphotericin because of severe sepsis. Tumour lysis syndrome occurs in fewer than $5 \%$ of children with ALL or T cell NHL and up to $15 \%$ of children with advanced B cell NHL; abnormal renal function may necessitate dialysis in these cases. ${ }^{2}{ }^{3}$ Severe febrile neutropenia in the 
early phases of treatment for ALL that requires amphotericin treatment occurs in $3-5 \%$ of children, ${ }^{3}$ mainly following the first intensification block, but is more common in infants who are especially vulnerable and who receive more intensive induction treatment. Children with these complications should be selected for a GFR before high dose MTX.
1 Bleyer WA. The clinical pharmacology of methotrexate. Cancer 1978;41:36-51.

2 Atra A, Gerrard M, Hobson R, Imeson JD, Ashley S, Pinkerton CR. Improved cure rate in children with B-cell acute ymphoblastic leukaemia (B-ALL) and stage IV B-cell non-Hodgkin's lymphoma (B-NHL) - results of the UKCCSG 9003 protocol. Br 7 Cancer 1998;77:2281-5.

3 Chessells JM, Bailey C, Richards SM. Intensification of treatment and survival in all children with lymphoblastic leukaemia: results of UK Medical Research Council trial UKALL X. Lancet 1995;345:143-8. 